\title{
Effects of Perinatal Fluoride Exposure on Short- and Long-Term Memory, Brain Antioxidant Status, and Glutamate Metabolism of Young Rat Pups
}

International Journal of Toxicology $1-10$

(C) The Author(s) 2019

Article reuse guidelines:

sagepub.com/journals-permissions DOI: $10.1|77 / 109158| 8 \mid 9857558$ journals.sagepub.com/home/ijt

@SAGE

\author{
Mariana Bartos' ${ }^{(}$, Fernanda Gumilar' ${ }^{(}$, , Cristina E. Gallegos', \\ Cristina Bras', Sergio Dominguez', Liliana M. Cancela², \\ and Alejandra Minetti ${ }^{\prime}$
}

\begin{abstract}
Exposure to fluoride $(F)$ during the development affects central nervous system of the offspring rats which results in the impairment of cognitive functions. However, the exact mechanisms of $F$ neurotoxicity are not clearly defined. To investigate the effects of perinatal $\mathrm{F}$ exposure on memory ability of young rat offspring, dams were exposed to 5 and $10 \mathrm{mg} / \mathrm{L} F$ during gestation and lactation. Additionally, we evaluated the possible underlying neurotoxic mechanisms implicated. The results showed that the memory ability declined in 45-day-old offspring, together with a decrease of catalase and glutamate transaminases activity in specific brain areas. The present study reveals that exposure to $F$ in early stages of rat development leads to impairment of memory in young offspring, highlighting the alterations of oxidative stress markers as well as the activity of enzymes involved in the glutamatergic system as a possible mechanisms of neurotoxicity.
\end{abstract}

\section{Keywords}

fluoride, perinatal exposure, memory, oxidative stress, glutamate metabolism

\section{Introduction}

Fluoride $(\mathrm{F})$ is a common element in nature and our daily life given that it is present in drinking water, soil, and the atmosphere. ${ }^{1,2}$ The main source of $\mathrm{F}$ for humans is the intake of groundwater contaminated by geological sources. ${ }^{3}$ Though groundwater contributes only $0.6 \%$ of the total water resources on earth, it is the major and preferred source of drinking water in rural as well as urban areas. ${ }^{4}$ It has been found that Argentina, ${ }^{5}$ as well as several countries in the world, has areas with shallow groundwater containing high $\mathrm{F}$ concentrations (up to $50 \mathrm{mg} / \mathrm{L}){ }^{6}$ Studies conducted in groundwater of the ChacoPampean plain (Argentina) show that most of the aquifers have average levels of 5 to $10 \mathrm{mg} / \mathrm{L} \mathrm{F}{ }^{7}$ In addition to drinking water, there are other sources of $\mathrm{F}$ like food contaminated with high concentrations of this element, dental products (toothpaste, mouth rinses), drugs, $\mathrm{F}$ dust, and fumes from industrial belt. ${ }^{2,8}$ Fluoride may be beneficial or detrimental depending on its concentration and total amount ingested. Fluoride is favorable especially for young children for calcification of dental enamel ${ }^{9}$; according to the World Health Organization, water supplementation with $\mathrm{F}$ for promoting dental health is usually in the concentration range of 0.5 to $1.0 \mathrm{mg} / \mathrm{L} .{ }^{10}$ It has been found that repeated ingestion of $\mathrm{F}(>1.5 \mathrm{mg} / \mathrm{L})$ over a prolonged period can result in toxic effects (fluorosis) in humans and animals. ${ }^{11}$ The most recent controversial aspects of $\mathrm{F}$ are related to toxicity of the developing brain ${ }^{12}$ and how it may possibly result in the decrease of intelligence quotient (IQ) and disturbances of learning and memory processes. ${ }^{13-16}$ It could also be involved in the etiology of other neurological disorders such as autism, behavioral fluctuations, fatigue, dizziness, vertigo, loss of motor coordination, complex paralysis of arms and legs, ${ }^{4,17}$ and neurodegenerative diseases such as Alzheimer and Parkinson. ${ }^{2,18}$

As reported previously, approximately $99 \%$ of the total body $\mathrm{F}$ is retained in bones and teeth, and the remainder can cross the cell membrane and be distributed in various soft tissues, such as the nervous system, liver, kidneys, reproductive system, skin, and erythrocytes. ${ }^{19}$ Fluoride passes through the

\footnotetext{
' Toxicology Lab. INBIOSUR, Departamento de Biología, Bioquímica y Farmacia, Universidad Nacional del Sur-CONICET, Buenos Aires, Argentina ${ }^{2}$ IFEC, Departamento de Farmacología, Universidad Nacional de Córdoba-CONICET, Córdoba, Argentina
}

\section{Corresponding Author:}

Mariana Bartos, Toxicology Lab. INBIOSUR, Departamento de Biología, Bioquímica y Farmacia, Universidad Nacional del Sur-CONICET, 8000 Bahía Blanca, Buenos Aires, Argentina.

Email: mbartos@criba.edu.ar 
placenta and its presence appear in low concentrations in saliva, sweat, and milk. ${ }^{1,20,21}$ It has been shown that F can penetrate the blood-brain barrier and accumulate in different regions of the brain, ${ }^{22,23}$ resulting in diverse lesions including demyelination, decrease in number of Purkinje cells, damage to the neurons and neuroglial cells, thickening and disappearance of dendrites, swelling of Nissl substance, and pyknosis of individual neurons. ${ }^{24}$ The neurotoxic effects of $\mathrm{F}$ (studies carried out with concentrations of $F$ ranging from 1 to $120 \mathrm{mg} / \mathrm{L}$ ) in the rat brain include oxidative stress, DNA damage, disturbance of protein and neurotransmitter levels, and alteration in the activities of some enzymes. ${ }^{25,26}$ Oxidative stress, one of the accepted mechanisms of $\mathrm{F}$ toxicity, is triggered by the imbalance between production and elimination of free radicals. ${ }^{16}$ When the body antioxidant capacity can no longer protect the cell from oxidative damage, free radicals such as reactive oxygen species (ROS) exert detrimental effects that can ultimately lead to changes in cell structure and function. ${ }^{27}$

Although F provides dental benefits when in drinking water at concentration of $0.7 \mathrm{mg} / \mathrm{L}$, epidemiological studies of populations exposed to greater than $1.5 \mathrm{mg} / \mathrm{L}$ have reported lower child intelligence and impairments in cognitive and neurobehavioral function, including learning and memory. ${ }^{28-31}$ Fluoride can alter the levels of some neurotransmitters and the activity of neurotransmitter metabolism-related enzymes. ${ }^{32}$ However, evidence about the effects of $F$ on the glutamate (Glu) neurotransmitter system still remains scarce. Glutamate is the main excitatory neurotransmitter in the mammalian central nervous system (CNS) and it plays an important role through ionotropic and metabotropic Glu receptors in learning and memory processes. ${ }^{33,34}$ This amino acid is supplied to the organism with the diet. However, only a small amount of Glu passes through the blood-brain barrier. This is a protective mechanism against excessive inflow of this neurotransmitter to the brain which could cause the depolarization and damaging of neurons. ${ }^{26}$ In addition, several mechanisms and enzymatic pathways are activated in order to maintain adequate extracellular Glu levels once this neurotransmitter is released into the synaptic cleft. ${ }^{35}$ With regard to the impact of $\mathrm{F}$ on brain's Glu metabolism, it has been reported that exposure of rodents to $\mathrm{F}$ through drinking water causes decrease in Glu level in cortex ${ }^{32}$ and hippocampus, ${ }^{26,32}$ impairment in the activities of Glu metabolism-related enzymes (including glutamic acid decarboxylase [GAD], glutamate oxaloacetate [GOT], and glutamate pyruvate [GPT] transaminases) in hippocampus ${ }^{26}$ together with impairment of learning and memory ability. ${ }^{26,32,36,37}$

Toxic effects of any environmental pollutant depend on the concentration and duration of its exposure as well as the susceptibility of the tissue to that particular toxicant. Maternal $\mathrm{F}$ exposure threatens the development of fetus. ${ }^{38}$ The CNS during development is highly sensitive to the influence of $\mathrm{F}$ due to its weak protective mechanisms. ${ }^{1}$ Therefore, the present study was designed to investigate the toxic effects of $F$ on memory ability and to detect alterations of the brain antioxidant status and Glu transaminases activity in young rat offspring treated with $F$ during embryonic and suckling stages (from gestational day 1 [GD 1]) to postnatal day 21 [PND21]). To this end, Wistar rats were exposed to $F$ concentrations $(5$ and $10 \mathrm{mg} / \mathrm{L}$ ) during gestation and lactation. In 45-day-old offspring, short-term memory (STM) and long-term memory (LTM) were evaluated by step-down inhibitory avoidance test; antioxidant enzymes such as catalase (CAT) and glutathione peroxidase (GPx), along with lipid peroxidation products such as malondialdehyde (MDA), were studied as potential biomarkers of oxidative stress; and transaminases (GOT and GPT) were assessed in specific brain areas (prefrontal cortex, striatum, and hippocampus), which are related to the neurobehavioral disorders observed in previous studies. ${ }^{39}$

\section{Materials and Methods}

\section{Materials}

Sodium fluoride (NaF; CAS No. 7681-49-4) was purchased from Anedra (San Fernando, Argentina). Transaminases kits were kindly provided by Wiener Lab (Rosario, Argentina).

\section{Animals and Experimental Design}

Male and nulliparous female Wistar rats (90-120 days old) were obtained from colonies maintained under specific pathogen-free conditions from our breeding center of the Universidad Nacional del Sur, Bahía Blanca, Argentina. They were maintained under constant temperature $\left(22^{\circ} \mathrm{C} \pm 1^{\circ} \mathrm{C}\right)$ and humidity (50\%-60\%) conditions in a 12 light:12 dark cycle (lights on at 7:00 AM) and with standard rodent pellet diet and filtered tap water ad libitum (the tap water contains less than $0.5 \mathrm{mg} / \mathrm{L} \mathrm{F}$ ). Female rats in proestrus stage were housed overnight with the male ones. The presence of spermatozoa in the vaginal smears was registered as an index of pregnancy and it was referred to as GD 0. Pregnant females were housed individually in cages and were randomly assigned to one of the 3 following groups: control group $(n=10$; filtered tap water), F-treated group with $5 \mathrm{mg} / \mathrm{L}$ in filtered tap water $(\mathrm{n}=10)$, and F-treated group with $10 \mathrm{mg} / \mathrm{L}$ in filtered tap water $(\mathrm{n}=10)$, equivalent to doses of 0.6 and $1.2 \mathrm{mg} / \mathrm{kg}$, respectively. Tap water was filtered using a Rotoplas water filter (Tusta, Buenas Aires, Argentina). This standard filter is responsible for retaining $99 \%$ of the particles (with a size equal to or greater than 50 $\mu \mathrm{m}$ ). Drinking water was changed daily. Dams received the treatment from GD 0 to weaning on PND21 (see scheme in Figure 1). Maternal weight gain, food intake, and drink consumption were recorded as described before. ${ }^{39,40}$. Additionally, all pups were weighed on different PNDs as described in Bartos et al. ${ }^{39,40}$ After weaning (PND21), offspring were housed according to sex and treatment, receiving filtered tap water and food ad libitum until PND45. One female and one male from each litter were randomly selected for the behavioral test and another 2 (of both sexes) from each litter were selected to perform neurochemical determinations in whole brain tissue and the specific brain areas: the prefrontal cortex, the striatum, 


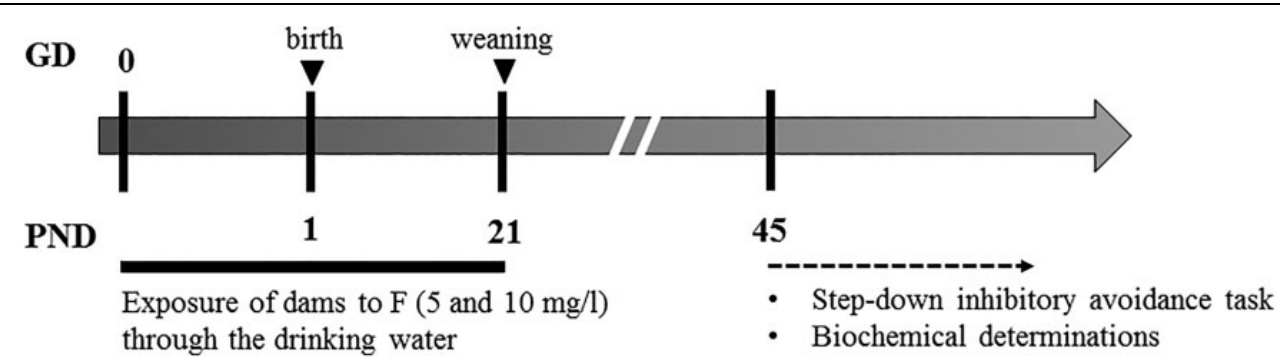

Figure I. Diagram of the experimental design.

and the hippocampus. Sample size used in memory test was $n$ $=9$ to 10 , whereas for neurochemical determinations, it was $n$ $=5$.

Animal care and handling were in accordance with the internationally accepted standard Guide for the Care and Use of Laboratory Animals ${ }^{41}$ as adopted and promulgated by the National Institute of Health. Experimental design was approved by Institutional Committee for the Care and Use of Experimental Animals of Universidad Nacional del Sur, Argentina. Number of animal protocol 021/2014.

\section{Step-Down Inhibitory Avoidance Task}

The PND45 offspring randomly selected for the behavioral test were trained in the step-down inhibitory avoidance paradigm. This is a trial that evaluates the hippocampal-dependent learning task in which stepping down from a platform presented in a given context is associated with a foot shock resulting in an increase in step-down latency. ${ }^{42}$ The inhibitory avoidance apparatus was a box with a floor consisting of parallel nonrusting steel bars. A 2.5-cm-high platform was placed on the left end of the box. Latency of the rats to step down placing the 4 paws on the grid was measured. Twenty-four hours prior to training, the rats were habituated to the new environment by placing them on the platform and allowing them to explore freely for 180 seconds. In the training session, the PND45 offspring were gently placed on the platform and received a $0.6 \mathrm{~mA}$ foot shock for 2 seconds after they stepped down the platform and placed their 4 paws on the grid. Test sessions were carried out for 1.5 hours (PND45) and 24 hours (PND46) after training for STM and LTM evaluations, respectively. ${ }^{42-44}$ They were exactly like the training session, except that the foot shock was omitted. A 180 second ceiling was imposed on test session latency measurements. In the test sessions, step-down latency was used as measure of retention of memory. ${ }^{45,46}$

\section{Preparation of Brain Homogenates}

Animals randomly selected for neurochemical determinations were sacrificed by decapitation on PND45. Brains were immediately removed from heads, rinsed in ice-cold phosphatebuffered saline (PBS; pH 7.4), and preserved as a whole or separated into prefrontal cortex, striatus, and hippocampus using an acrylic coronal brain matrix (Stoelting Co, Illinois) and the atlas of Paxinos and Watson ${ }^{47}$ as a guide for tissue dissection. The whole brain was homogenized with a dounce homogenizer, and the different sections were homogenized with disposable homogenization pestles. Then the homogenates were centrifuged and the supernatants were kept cold until the determinations of enzymes activities and lipid peroxidation level were made.

\section{Thiobarbituric Acid Reactive Substance Determination}

As an indicator of lipid peroxidation level present in the whole brain, MDA was assayed as thiobarbituric acid reactive substance. ${ }^{48}$ Briefly, $1 \mathrm{~mL}$ of whole brain supernatant of PND45 offspring was mixed with $1 \mathrm{~mL} 20 \%$ trichloroacetic acid (TCA), followed by 30-minute incubation on ice. After centrifugation at 3,000 rpm for 10 minutes, $1 \mathrm{~mL}$ of the TCA supernatant was incubated with $1 \mathrm{~mL}$ of a $0.8 \%$ thiobarbituric acid solution for 30 minutes at $100^{\circ} \mathrm{C}$. Samples were allowed to cool at room temperature, and absorbance was measured spectrophotometrically at $532 \mathrm{~nm}$. Malondialdehyde concentrations were determined by using an acid hydrolysis product of 1,1,3,3-tetraethoxypropane as a standard. Malondialdehyde concentration was expressed as nanomole per milligram protein.

\section{Antioxidant Enzyme Activities}

Activity of CAT and GPx was determined in 45-day-old offspring. The CAT activity of the whole brain and the different sections was assayed following the procedure of $\mathrm{Aebi}^{49}$ with slight modifications. Reaction was initiated by addition of 0.5 $\mathrm{mL} \mathrm{H}_{2} \mathrm{O}_{2}$ (1/10 in PBS) to the reaction mixture containing 100 $\mu \mathrm{L}$ supernatant, $25 \mu \mathrm{L}$ Triton-X 100 (1/10 in PBS), and $2.4 \mathrm{~mL}$ PBS. The decrease in absorbance was recorded for 3 minutes at $240 \mathrm{~nm}$. The enzyme activity was expressed as the rate constant of a first-order reaction $(\mathrm{k})$ per $\mathrm{mg}$ protein.

The GPx activity of the whole brain was measured by the method used by Lawrence and Burk. ${ }^{50}$ Reaction medium containing $100 \mu \mathrm{L}$ Glutathione reduced $(10 \mathrm{mM}), 100 \mu \mathrm{L}$ glutathione reductase ( $0.24 \mathrm{U}$ of enzyme activity), $10 \mu \mathrm{L}$ of sodium azide $(100 \mathrm{mM})$, and $20 \mu \mathrm{L}$ of supernatant was incubated at $37^{\circ} \mathrm{C}$ for 10 minutes. Then $100 \mu \mathrm{L}$ of Nicotinamide adenine dinucleotide phosphate (NADPH) $(3 \mathrm{mM})$ was added, followed by the rapid addition of $100 \mu \mathrm{L}$ of $\mathrm{H}_{2} \mathrm{O}_{2}(2 \mathrm{mM})$. The 


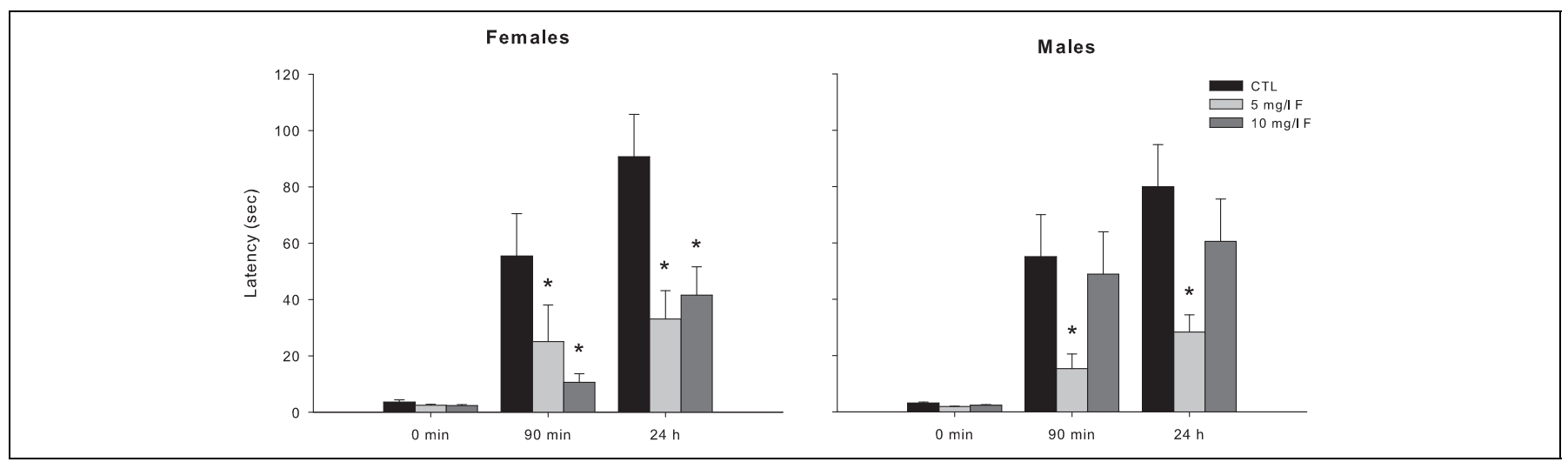

Figure 2. Latencies measured in the step-down inhibitory avoidance test during the training session $(0$ minutes $)$ and the test sessions for the evaluation of STM ( 90 minutes after training) and LTM (24 hours after training) in the young female and male offspring exposed to 5 and I 0 mg/L F. Data represent the mean \pm SEM latency in seconds of 9 to 10 animals per group. $* P<0.05$, compared to control group. SEM indicates standard error of the mean; SRM, short-term memory; LTM, long-term memory.

decrease in absorbance was recorded for 3 minutes at $340 \mathrm{~nm}$. The enzyme activity was expressed as micromoles de NADPH oxidized per minute per mg protein.

\section{Glutamate Transaminases}

Activity of GOT and GPT transaminases was evaluated in homogenates of brain areas of PND45 offspring exposed to both $\mathrm{F}$ concentrations, by spectrophotometric methods using the corresponding commercial kits of Wiener Lab and following the manufacturers' indications.

\section{Protein Assay}

Protein concentration of the supernatants was measured using the micromethod of Bradford. ${ }^{51}$ Bovine serum albumin was used as a standard.

\section{Statistics}

Since step-down latencies are data that do not follow a normal distribution, they were statistically analyzed by the nonparametric Kruskal-Wallis test followed by Mann-Whitney $U$ test to compare between groups. This analysis was carried out considering data from separated sexes in each session of the step-down inhibitory avoidance test (training, 90 minutes and 24 hours).

Data of enzyme activities and lipid peroxidation level from the total brain homogenate and the different brain region homogenates were analyzed by 2-way analysis of variance (ANOVA), considering dose groups (control, $5 \mathrm{mg} / \mathrm{L} \mathrm{F}$, and $10 \mathrm{mg} / \mathrm{L} \mathrm{F}$ ) and sexes as factors. Differences between groups were assessed using least significant difference post hoc test.

Probability values lower than 0.05 were considered to be significant. All statistical analyses were carried out using software SPSS 21.0 for Windows.

\section{Results}

\section{Data About the Dams and Their Litters}

There were no statistical differences detected in body weight and water and food intake among the groups of dams (data not shown). Furthermore, F exposure during gestation and lactation did not significantly affect the body weight of female and male pups on different PNDs $(1,4,7,10,13,16,19,21$, and 45; data not shown). No visible teratogenic malformations were observed in any of the groups tested. Similar results were obtained and detailed previously by Bartos et al. ${ }^{39,40}$

\section{Step-Down Inhibitory Avoidance Test}

To evaluate the STM and LTM, we performed the inhibitory avoidance test on PND45 offspring exposed during gestation and lactation to 5 or $10 \mathrm{mg} / \mathrm{L} \mathrm{F}$. The data represented in Figure 2 show the results of the step-down inhibitory avoidance test.

In female 45-days-old offspring, the exposure to 5 and 10 $\mathrm{mg} / \mathrm{L} \mathrm{F}$ produced a significant impairment in the retention of both STM and LTM. The latencies of these rats to step down on the grid were lower than those of the control group 90 minutes and 24 hours after training $(H=9.89, P<0.05$ and $H=6.52$, $P<0.05$, respectively, for each time session; Figure 2). Latency of male rats exposed to $\mathrm{F}$ during gestation and lactation was statistically different in both STM and LTM retention $(H=$ 6.34, $P<0.05$ and $H=6.11, P<0.05$, respectively; Figure 2). Post hoc comparisons showed that latencies of males exposed to $5 \mathrm{mg} / \mathrm{L} \mathrm{F}$ were shorter than the control group in both STM and LTM. However, latency of male exposed to $10 \mathrm{mg} / \mathrm{L} \mathrm{F}$ did not differ from that of control at the 2 retention tests. The latencies measured in the training session were similar for all groups.

\section{Oxidative Stress in the Brains of PND45 Offspring}

Alteration of biochemical markers of oxidative damage was observed in whole brain and in the different brain section 

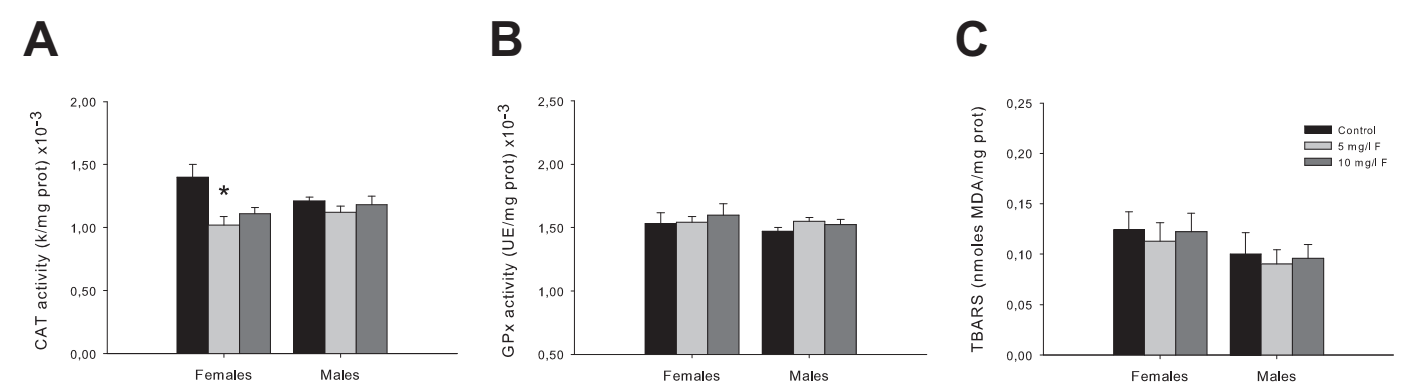

Figure 3. Activities of CAT (A) and GPx (B), and MDA content (C) in the whole brain homogenates of 45-day-old female and male offspring exposed to 5 and $10 \mathrm{mg} / \mathrm{L} F$ during the gestation and lactation. The values are shown as the means \pm SEM of 5 rats per group. $* P<0.05$, compared to control group. CAT indicates catalase; GPx, glutathione peroxidase; MDA, malondialdehyde; SEM, standard error of the mean.

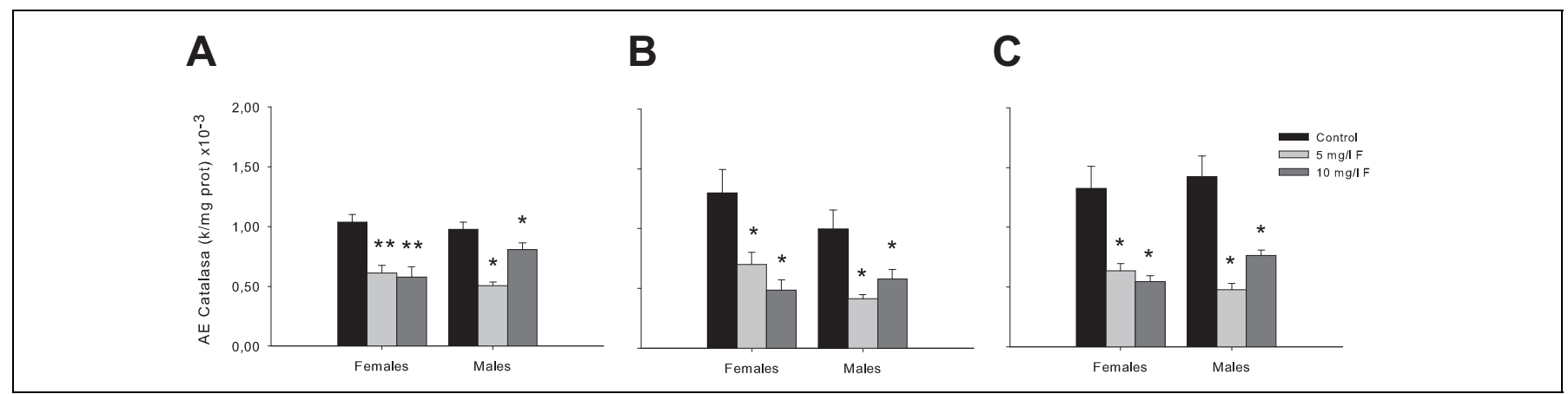

Figure 4. Activities of CAT in the prefrontal cortex (A), the striatum (B), and the hippocampus (C) of PND45 offspring exposed to 5 and $10 \mathrm{mg} / \mathrm{L} \mathrm{F}$. The values are shown as the means \pm SEM of 5 rats per group. $* P<0.05$ and $* * P<0.00$ I, compared to control group. CAT indicates catalase; PND, postnatal day; SEM, standard error of the mean.

homogenates of PND45 offspring exposed to F during gestation and lactation. Figure 3 illustrates the activities of antioxidant enzymes, namely CAT and GPx, and levels of lipid peroxidation as MDA content, in the whole brain of control and experimental groups of young female and male offspring exposed to 5 and $10 \mathrm{mg} / \mathrm{L}$ F during development.

Catalase activity results analyzed by 2 -way ANOVA showed significant differences between groups $\left(F_{2,24}=6.14\right.$; $P<0.05)$. Post hoc comparisons showed that female PND45 rats from $5 \mathrm{mg} / \mathrm{L}$ F-treated group exhibited a significant decrease in this parameter compared to the corresponding control group $(P<0.05$; Figure $3 \mathrm{~A})$. No significant differences were observed in 2-way ANOVA in GPx activity or MDA levels (Figure 3B and C).

We subsequently continued the analysis of oxidative stress in the prefrontal cortex, the striatum, and the hippocampus. Here, we only analyzed CAT activity since it showed a statistically significant reduction in the whole brain homogenate (Figure 3A). Two-way ANOVA revealed significant differences between groups in the activity of this enzyme in the prefrontal cortex $\left(F_{2,30}=28.03 ; P<0.001\right.$; Figure $\left.4 \mathrm{~A}\right)$, the striatum $\left(F_{2,30}=11.12 ; P<0.001 ;\right.$ Figure $\left.4 \mathrm{~B}\right)$, and the hippocampus $\left(F_{2,30}=12.77 ; P<0.001\right.$; Figure $\left.4 \mathrm{C}\right)$. Post hoc comparisons showed a significant decrease in females as well as males from both $\mathrm{F}$ concentrations $(5$ and $10 \mathrm{mg} / \mathrm{L} \mathrm{F})$ tested in the prefrontal cortex $(P<0.001$ and $P<0.05$ for females and males, respectively; Figure 4A), in the striatum $(P<0.05$ for both sexes; Figure 4B), and in the hippocampus $(P<0.05$ for all PND45 offspring; Figure 4C) compared to the respective controls.

\section{Evaluation of the Glu Transaminase Activity}

Glu transaminases GPT and GOT are known regulators of the metabolism of the excitatory neurotransmitter Glu. ${ }^{35,52}$ In order to investigate if $\mathrm{F}$ exposure during the gestational and lactation periods affects Glu metabolism, the activity of GPT and GOT was determined in specific brain areas of young female and male offspring exposed to 5 and $10 \mathrm{mg} / \mathrm{L} \mathrm{F}$. As show in Figure 5, transaminases activities in the prefrontal cortex, the striatum, and the hippocampus of control and treated groups were assessed.

Two-way ANOVA analysis resulted in significant differences between groups in each of the brain areas studied, both for GPT (prefrontal cortex: $F_{2,28}=21.79 ; P<0.001$; striatum: $F_{2,25}=14.37 ; P<0.001$; hippocampus: $F_{2,26}=7.53 ; P<$ 0.05 ) and GOT (prefrontal cortex: $F_{2,26}=60.92 ; P<0.001$; striatum: $F_{2,27}=60.73 ; P<0.001$; hippocampus: $F_{2,27}=$ 38.55; $P<0.001)$.

Post hoc tests showed that in female PND45 offspring, the activity of GPT was significantly decreased in prefrontal cortex and hippocampus for both $\mathrm{F}$ concentration tested compared to 

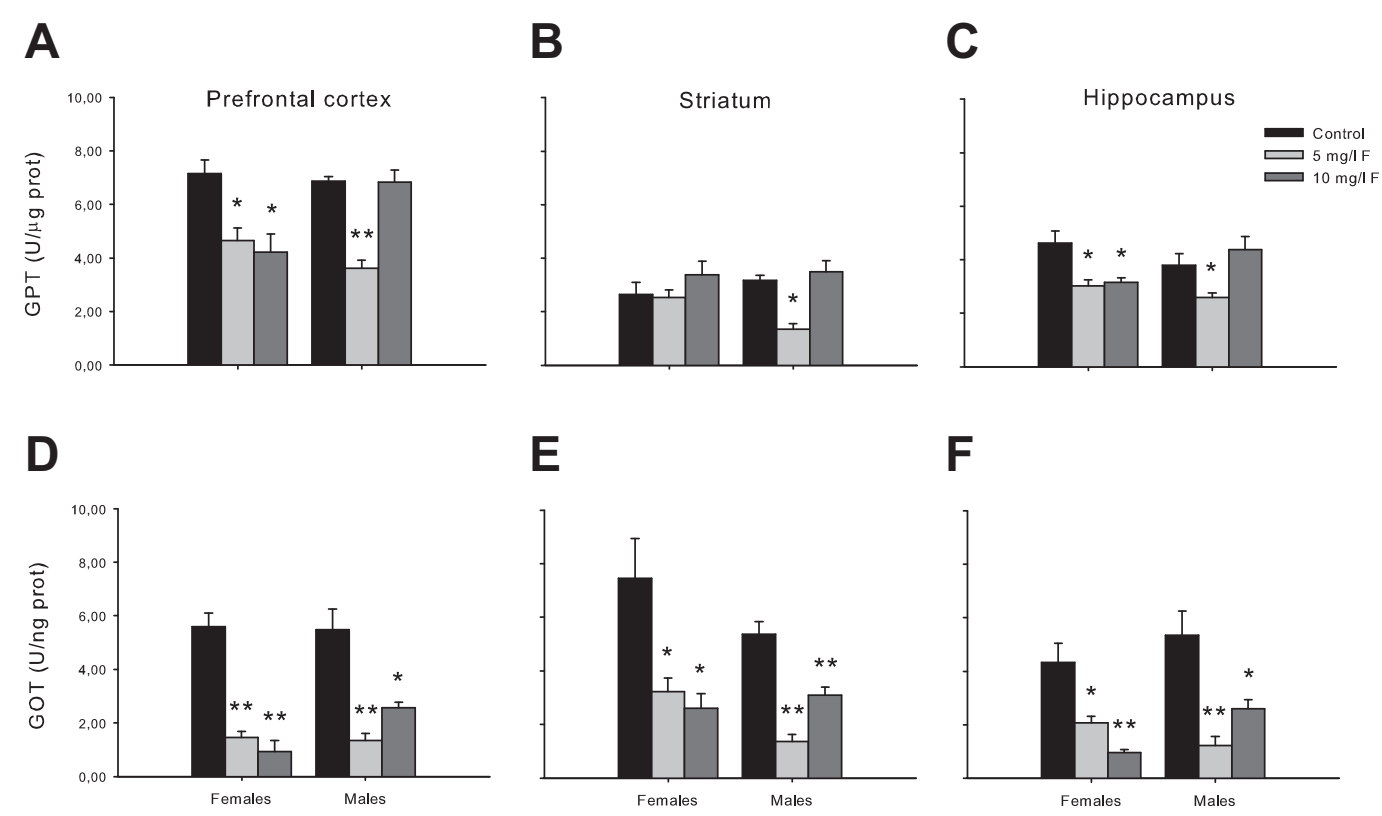

Figure 5. Transaminase activities (GPT and GOT) in the prefrontal cortex, the striatum, and the hippocampus of 45-days-old offspring. Data are expressed as mean \pm SEM. $n=5$ per group. $* P<0.05 ; * * P<0.001$ compared to the corresponding control group. GOT indicates glutamate oxaloacetate; GPT, glutamate pyruvate; SEM, standard error of the mean.

control groups (Figure 5A an C), with exception of striatum in which GPT activity did not evidence any change (Figure 5B). In young male rats, we observed a similar tendency to decrease in all brain areas, but only for the lowest $\mathrm{F}$ concentration tested (Figure 5A-C).

In the GOT activity, post hoc comparisons showed a significant decrease in female offspring from both $\mathrm{F}$ concentrations ( 5 and $10 \mathrm{mg} / \mathrm{L} \mathrm{F}$ ) tested in the prefrontal cortex (Figure 5D), the striatum (Figure 5E), and the hippocampus (Figure 5F) compared to the respective controls. In male 45 -day-old offspring, also both F concentration tested ( 5 and $10 \mathrm{mg} / \mathrm{L} \mathrm{F}$ ) produced largest rate decrease in the GOT activity in all brain sections (Figure 5D-F).

\section{Discussion}

Numerous studies on F metabolism confirmed that it can penetrate the placenta and it may adversely affect the development of fetal nervous system. ${ }^{2}$ The exposure continues during lactation by the passage of $\mathrm{F}$ through maternal milk. ${ }^{53}$ Therefore, the model of maternal $\mathrm{F}$ exposure during gestation and lactation (from GD0 to PND21) was established in this study to investigate the neurotoxic effects of $F$ on young pups.

We here demonstrated that early exposure to $\mathrm{F}$ impairs the retention of memory in young offspring rats evaluated in the step-down inhibitory avoidance task. Our results demonstrate that $5 \mathrm{mg} / \mathrm{L} \mathrm{F}$ significantly impaired memory of 90 minutes (STM) and 24 hours (LTM) in both female and male PND45 offspring, whereas exposure to $10 \mathrm{mg} / \mathrm{L} \mathrm{F}$ only affected STM and LTM in female pups. Emotionally arousing experiences create LTMs that are initially labile, but over time become insensitive to disruption through a process known as consolidation. ${ }^{54}$ In this respect, step-down inhibitory avoidance test is a one-trial fear-motivated learning task, a hippocampaldependent associative learning, ${ }^{46}$ that has largely contributed to the knowledge of consolidation process. The memory process impairment observed in PND45 offspring exposed early to $\mathrm{F}$ and evaluated by the step-down inhibitory avoidance task is an effect that might be related to the cognitive and memory alterations in people exposed to $\mathrm{F}$ in drinking water. Epidemiological studies conducted in children exposed to $\mathrm{F}$ have shown deficits in IQ and reduced mental ability. The IQ scores declined with increasing $\mathrm{F}$ exposure. ${ }^{15}$ It has been suggested that $\mathrm{F}$ cause harmful effects on the CNS by negatively affecting both cognitive and psychomotor functions. ${ }^{11}$ Perinatal $\mathrm{F}$ exposure $(25-100 \mathrm{mg} / \mathrm{L})$ reduces the myelinated nerve fibers and alter the DNA structure in the hippocampus of mouse pups. ${ }^{55}$ Fluoride exposure $(2-10 \mathrm{mg} / \mathrm{L})$ through drinking water caused cognitive impairment and led to anxiety and depression-like behavior in adult mice. ${ }^{56}$

Studies on both human and animals chronically exposed to $\mathrm{F}$ (60 and $120 \mathrm{mg} / \mathrm{L}$ ) indicated vulnerability of all soft tissue organs. ${ }^{57}$ Additionally, the CNS is much more susceptible to toxic agents in the early stages of growth and development. The embryonic period in humans and rodents is highly sensitive to chemical toxicity. ${ }^{58}$ The brain and neural cells are highly susceptible to oxidative damage as they contain high amount of polyunsaturated fatty acids, rich iron content, and low levels of antioxidant enzymes. ${ }^{59}$ As a free radical inducer, F (600 mg/L) produces excessive ROS and causes oxidative damage in the rat brain which is a causative factor for the cognitive impairment. ${ }^{60}$ Free radicals cause toxicity by attacking membrane 
phospholipids, leading to membrane injury through lipid peroxidation, depolarization of the mitochondrial membrane, and apoptosis. ${ }^{3,12}$

In the present study, we analyzed the activities of the antioxidant enzymes CAT and GPx, together with the determination of MDA content in the complete brain of control and experimental groups of PND45 offspring. Since in whole brain determinations we only detected statistical significance in the activity of the enzyme CAT, we subsequently continued the analysis of oxidative stress in the different brain areas only for this antioxidant enzyme. As can be observed, the results of CAT activity in the prefrontal cortex, the striatum, and the hippocampus were more noticeable than those found in whole brain homogenates. The explanation for this phenomenon might be that when we are analyzing the activity of an enzyme in the totality of the brain tissue, changes in a punctual area could be masked by the nonchanges in the remaining areas. Our results showed that maternal exposure to $\mathrm{F}$ during pregnancy and lactation decreased the enzymatic activity of CAT in the prefrontal cortex, the striatum, and the hippocampus. In this respect, it is important to denote that both an increase and/or a decrease in the expression or activity of antioxidant enzymes are indicative of oxidative stress. ${ }^{61}$ These brain areas are critical in the regulation of locomotion, anxiety, and memory, and their regulatory functions are usually overlapped. Thus, the prefrontal cortex is implicated in the control of locomotor activity, the regulation of emotional states, and cognitive processes such as working memory. ${ }^{62}$ Locomotor activity is also regulated by the striatum. ${ }^{63}$ In addition, this brain area has been recently included to the anxiety network, integrated also by hippocampus and prefrontal cortex. ${ }^{64}$ The hippocampus is well known as a key region of the limbic system that is crucial for intellectual function, cognitive ability, and anxiety. ${ }^{65,66}$ In a previous work from our laboratory, we demonstrated that the oral exposure of rats to $\mathrm{F}$ during gestation and lactation decreased the anxiety levels in female 45-day-old offspring. ${ }^{39}$

As we observed that $\mathrm{F}$ exposure impairs the retention of memory of PND45 offspring rats, and considering that the excitatory neurotransmitter Glu plays an important role in learning and memory processes, ${ }^{33,34}$ we decide to evaluate if the glutamatergic system was altered by $\mathrm{F}$ treatment. For achieving this purpose, we assessed the activities of transaminases GPT and GOT in prefrontal cortex, striatum, and hippocampus of young offspring exposed to $\mathrm{F}$ during gestation and lactation. Our results showed that exposure to $\mathrm{F}$ in early stages of rat development decreased the activity of both transaminases in specific brain areas of 45-day-old offspring. Neuronal GOT and GPT are active brain enzymes involved in Glu metabolism, ${ }^{67}$ and the reduction of its activity might impact on the levels of this neurotransmitter. These transaminases catalyze the transfer of an amino group from aspartate (GOT) or from alanine (GPT) to $\alpha$-ketoglutarate, to produce Glu and oxaloacetate (in the case of GOT) or Glu and pyruvate (in the case of GTP) ${ }^{67}$ The opposite reaction (ie, oxidation of Glu) is also possible, since for both enzymes the reaction is freely reversible. ${ }^{35,52,67}$ In this regard, Niu et al. ${ }^{26}$ reported that exposure of rats to $F(150 \mathrm{mg} / \mathrm{L})$ during lactation and after weaning significantly decreases learning abilities in the pups. In addition, this treatment caused increase in the activity of GAD and inhibition of the activity of GOT and GPT, leading to the concomitant decrease in the levels of Glu in the hippocampus of exposed rats. These authors suggested that the disturbance in Glu metabolism may be one of the mechanisms by which $\mathrm{F}$ influence intelligence. Similarly, Jiang et al. ${ }^{32}$ reported that exposure of rats to $\mathrm{F}(120 \mathrm{mg} / \mathrm{L})$ caused declination of learning and memory ability, together with a decrease in Glu levels in hippocampus and cortex of the exposed rats. On the other hand, Glu excitotoxicity has also been proposed as a possible mechanism of F neurotoxicity. ${ }^{68,69}$ The association between neurotoxicity with oxidative stress and Glu excitotoxicity has been widely reported. ${ }^{68,70,71}$ However, with the results obtained so far, it is difficult to propound a precise mechanism for F neurotoxicity. Other determinations, such as measurement of the levels of Glu in specific brain areas, or the activity of other Glu metabolism-related enzymes (besides GOT and GPT), would be necessary in order to complement the obtained data and to define if the glutamatergic system is involved.

It is important to highlight that in almost all the parameters analyzed in the present study, in PND45 male offspring, we could observe a trend of a greater response at $5 \mathrm{mg} / \mathrm{L}$, whereas for the higher dose $(10 \mathrm{mg} / \mathrm{L} \mathrm{F})$, the response was lesser or nonsignificant. We can speculate that there could be compensatory mechanisms that are triggered at a higher F concentration, which would avoid an impairment of memory in the step-down inhibitory avoidance test, an increase of oxidative stress, and a reduction in the transaminases activity. However, the detailed mechanism of why the lowest concentration of $F$ in male offspring exerts more deleterious effects than the higher concentration, of course, needs further investigation to find out a possible mechanism of $\mathrm{F}$ toxicity.

In summary, our findings indicate that exposure of rats to $\mathrm{F}$ through cord blood and breast milk resulted in the impairment of memory in 45-day-old female and male offspring. Although the levels of exposure in this study exceeded the concentrations to which $\mathrm{F}$ supplementation is generally implemented to promote good dental health $\left(0.5-1.0 \mathrm{mg} / \mathrm{L}^{10}\right)$, F levels in naturally occurring waters can include or exceed the exposure concentrations in this study. Among the possible mechanisms postulated to mediate these effects, the most plausible would be the induction of oxidative stress and the decreased activity of the enzymes GOT and GPT (which in turn could affect the metabolism of Glu) in specific brain areas of young offspring rats.

\section{Acknowledgments}

The authors thank Wiener Lab, Argentina, for the donation of transaminase kits.

\section{Author Contributions}

M.B. contributed to conception and design, contributed to acquisition, analysis, and interpretation, and drafted the manuscript. F.G. contributed to acquisition. C.G. contributed to acquisition and analysis and critically revised the manuscript. C.B. contributed to acquisition and 
International Journal of Toxicology $X X(X)$

critically revised the manuscript. S.D. contributed to acquisition. L.C. critically revised the manuscript. A.M. contributed to conception and design and critically revised the manuscript. All authors gave final approval and agree to be accountable for all aspects of work ensuring integrity and accuracy.

\section{Declaration of Conflicting Interests}

The author(s) declared no potential conflicts of interest with respect to the research, authorship, and/or publication of this article.

\section{Funding}

The author(s) disclosed receipt of the following financial support for the research, authorship, and/or publication of this article: This research was supported by grants from the Secretaria General de Ciencia y Tecnología from the Universidad Nacional del Sur (UNS; grant number PGI 24/B223), the National Research Council (CONICET; grant number PIP 11220130100393CO), and National Agency for the Promotion of Science and Technology of Argentina (ANPCyT; grant number PICT 2013-2012).

\section{ORCID iD}

Mariana Bartos (D) https://orcid.org/0000-0002-5677-4828

Fernanda Gumilar (D) https://orcid.org/0000-0002-7789-2647

\section{References}

1. Basha PM, Madhusudhan N. Pre and post natal exposure of fluoride induced oxidative macromolecular alterations in developing central nervous system of rat and amelioration by antioxidants. Neurochem Res. 2010;35(7):1017-1028.

2. Prystupa J. Fluorine - a current literature review. An NRC and ATSDR based review of safety standards for exposure to fluorine and fluorides. Toxicol Mech Method. 2011;21(2):103-170.

3. Barbier O, Arreola-Mendoza L, Del Razo LM. Molecular mechanisms of fluoride toxicity. Chem-Biol Interact. 2010; 188(2):319-333.

4. Sarkar C, Pal S. Ameliorative effect of resveratrol against fluoride-induced alteration of thyroid function in male Wistar rats. Biol Trace Elem Res. 2014;162(1-3):278-287.

5. Nicolli HB, Bundschuh J, Blanco Mdel C, et al. Arsenic and associated trace-elements in groundwater from the ChacoPampean plain, Argentina: results from 100 years of research. Sci Total Environ. 2012;429:36-56.

6. Amini M, Mueller K, Abbaspour KC, et al. Statistical modeling of global geogenic fluoride contamination in groundwaters. Environ Sci Technol. 2008;42(10):3662-3668.

7. Alarcon-Herrera MT, Bundschuh J, Nath B, et al. Co-occurrence of arsenic and fluoride in groundwater of semi-arid regions in Latin America: genesis, mobility and remediation. $J$ Hazard Mater. 2013;262:960-969.

8. Hassan HA, Yousef MI. Mitigating effects of antioxidant properties of black berry juice on sodium fluoride induced hepatotoxicity and oxidative stress in rats. Food Chem Toxicol. 2009;47(9): 2332-2237.

9. Ismail AI, Hasson H. Fluoride supplements, dental caries and fluorosis: a systematic review. J Am Dent Assoc. 2008;139(11): 1457-1468.
10. Guidelines for Drinking-Water Quality: Fourth Edition Incorporating the First Addendum. WHO Guidelines Approved by the Guidelines Review Committee. Geneva, Switzerland: World Health Organization; 2017.

11. Dec K, Lukomska A, Maciejewska D, et al. The influence of fluorine on the disturbances of homeostasis in the central nervous system. Biol Trace Elem Res. 2017;177(2):224-234.

12. Atmaca N, Atmaca HT, Kanici A, Anteplioglu T. Protective effect of resveratrol on sodium fluoride-induced oxidative stress, hepatotoxicity and neurotoxicity in rats. Food Chem Toxicol. 2014;70:191-197.

13. Chioca LR, Raupp IM, Da Cunha C, Losso EM, Andreatini R. Subchronic fluoride intake induces impairment in habituation and active avoidance tasks in rats. Eur J Pharmacol. 2008;579(1-3): 196-201.

14. Ranpariya VL, Parmar SK, Sheth NR, Chandrashekhar VM. Neuroprotective activity of Matricaria recutita against fluorideinduced stress in rats. Pharm Biol. 2011;49(7):696-701.

15. Choi AL, Sun G, Zhang Y, Grandjean P. Developmental fluoride neurotoxicity: a systematic review and meta-analysis. Environ Health Persp. 2012;120(10):1362-1368.

16. Banala RR, Karnati PR. Vitamin a deficiency: an oxidative stress marker in sodium fluoride $(\mathrm{NaF})$ induced oxidative damage in developing rat brain. Int J Dev Neurosci. 2015;47(Pt B):298-303.

17. Bera I, Sabatini R, Auteri P, et al. Neurofunctional effects of developmental sodium fluoride exposure in rats. Eur Rev Med Pharmacol Sci. 2007;11(4):211-224.

18. Hussien HM, Abd-Elmegied A, Ghareeb DA, Hafez HS, Ahmed HEA, El-Moneam NA. Neuroprotective effect of berberine against environmental heavy metals-induced neurotoxicity and Alzheimer's-like disease in rats. Food Chem Toxicol. 2018;111: 432-444.

19. Chouhan S, Flora SJ. Effects of fluoride on the tissue oxidative stress and apoptosis in rats: biochemical assays supported by IR spectroscopy data. Toxicology. 2008;254(1-2):61-67.

20. Fassman DK. Prenatal fluoridation. A literature review. N Y State Dent J. 1993;59(6):47-51.

21. Niu R, Xue X, Zhao Y, et al. Effects of fluoride on microtubule ultrastructure and expression of Tubalphala and Tubbeta2a in mouse hippocampus. Chemosphere. 2015;139:422-427.

22. Whitford GM, Whitford JL, Hobbs SH. Appetitive-based learning in rats: lack of effect of chronic exposure to fluoride. Neurotoxicol Teratol. 2009;31(4):210-215.

23. Wang C, Liang C, Ma J, et al. Co-exposure to fluoride and sulfur dioxide on histological alteration and DNA damage in rat brain. J Biochem Mol Toxicol. 2018;32(2):1-5.

24. Shashi A. Histopathological investigation of fluoride-induced neurotoxicity in rabbits. Fluoride 2003;36(95):95-105.

25. Flora SJ, Mittal M, Pachauri V, Dwivedi N. A possible mechanism for combined arsenic and fluoride induced cellular and DNA damage in mice. Metallomics. 2012;4(1):78-90.

26. Niu R, Sun Z, Cheng Z, Li Z, Wang J. Decreased learning ability and low hippocampus glutamate in offspring rats exposed to fluoride and lead. Environ Toxicol Pharmacol. 2009;28(2): 254-258. 
27. Nabavi SF, Nabavi SM, Habtemariam S, Moghaddam AH, Sureda A, Mirzaei M. Neuroprotective effects of methyl-3-O-methyl gallate against sodium fluoride-induced oxidative stress in the brain of rats. Cell Mol Neurobiol. 2013;33(2):261-267.

28. Rocha-Amador D, Navarro ME, Carrizales L, Morales R, Calderon J. Decreased intelligence in children and exposure to fluoride and arsenic in drinking water. Cad Saude Publica. 2007; 23(suppl 4):S579-S587.

29. Seraj B, Shahrabi M, Shadfar M, et al. Effect of high water fluoride concentration on the intellectual development of children in Makoo/Iran. J Dent. 2012;9(3):221-229.

30. Saxena S, Sahay A, Goel P. Effect of fluoride exposure on the intelligence of school children in Madhya Pradesh, India. J Neurosci Rural Pract. 2012;3(2):144-149.

31. Wang SX, Wang ZH, Cheng XT, et al. Arsenic and fluoride exposure in drinking water: children's IQ and growth in Shanyin county, Shanxi province, China. Environ Health Perspect. 2007; 115(4):643-647.

32. Jiang S, Su J, Yao S, et al. Fluoride and arsenic exposure impairs learning and memory and decreases mGluR5 expression in the hippocampus and cortex in rats. PloS One. 2014;9(4):e96041.

33. Dennis SH, Pasqui F, Colvin EM, et al. Activation of muscarinic M1 acetylcholine receptors induces long-term potentiation in the hippocampus. Cereb Cortex. 2016;26(1):414-426.

34. Myhrer T. Neurotransmitter systems involved in learning and memory in the rat: a meta-analysis based on studies of four behavioral tasks. Brain Res Brain Res Rev. 2003;41(2-3):268-287.

35. Daikhin Y, Yudkoff M. Compartmentation of brain glutamate metabolism in neurons and glia. J Nutr. 2000;130(suppl 4S): 1026S-10231S.

36. Ge Y, Chen L, Yin Z, et al. Fluoride-induced alterations of synapse-related proteins in the cerebral cortex of ICR offspring mouse brain. Chemosphere. 2018;201:874-883.

37. Sun Z, Zhang Y, Xue X, Niu R, Wang J. Maternal fluoride exposure during gestation and lactation decreased learning and memory ability, and glutamate receptor mRNA expressions of mouse pups. Hum Exp Toxicol. 2018;37(1):87-93.

38. Perumal E, Paul V, Govindarajan V, Panneerselvam L. A brief review on experimental fluorosis. Toxicol Lett. 2013;223(2): 236-251.

39. Bartos M, Gumilar F, Bras C, et al. Neurobehavioural effects of exposure to fluoride in the earliest stages of rat development. Physiol Behav. 2015;147:205-212.

40. Bartos M, Gumilar F, Gallegos CE, et al. Alterations in the memory of rat offspring exposed to low levels of fluoride during gestation and lactation: involvement of the alpha7 nicotinic receptor and oxidative stress. Reprod Toxicol. 2018;81:108-114.

41. The National Institute of Health. Guide for the Care and Use of Laboratory Animals. 8th ed. Bethesda, MD: The National Institute of Health; 2011.

42. Bevilaqua LR, Kerr DS, Medina JH, Izquierdo I, Cammarota M. Inhibition of hippocampal Jun N-terminal kinase enhances shortterm memory but blocks long-term memory formation and retrieval of an inhibitory avoidance task. Eur J Neurosci. 2003;17(4): 897-902.
43. Izquierdo I, Medina JH, Vianna MR, Izquierdo LA, Barros DM. Separate mechanisms for short- and long-term memory. Behav Brain Res. 1999;103(1):1-11.

44. Giovannini MG, Lana D, Pepeu G. The integrated role of ACh, ERK and mTOR in the mechanisms of hippocampal inhibitory avoidance memory. Neurobiol Learn Mem. 2015;119:18-33.

45. Canto-de-Souza L, Mattioli R. The consolidation of inhibitory avoidance memory in mice depends on the intensity of the aversive stimulus: the involvement of the amygdala, dorsal hippocampus and medial prefrontal cortex. Neurobiol Learn Mem. 2016;130:44-51.

46. Izquierdo I, Medina JH. Memory formation: the sequence of biochemical events in the hippocampus and its connection to activity in other brain structures. Neurobiol Learn Mem. 1997; 68(3):285-316.

47. Paxinos G, Watson C. The Rat Brain in Stereotaxic Coordinates. 6th ed. Elsevier, 2007.

48. Beuret CJ, Zirulnik F, Gimenez MS. Effect of the herbicide glyphosate on liver lipoperoxidation in pregnant rats and their fetuses. Reprod Toxicol. 2005;19(4):501-504.

49. Aebi H. Catalase in vitro. Methods Enzymol. 1984;105:121-126.

50. Lawrence RA, Burk RF. Glutathione peroxidase activity in selenium-deficient rat liver. Biochem Bioph Res Co. 1976; 71(4):952-958.

51. Bradford MM. A rapid and sensitive method for the quantitation of microgram quantities of protein utilizing the principle of protein-dye binding. Anal Biochem. 1976;72:248-254.

52. Matthews CC, Zielke HR, Wollack JB, Fishman PS. Enzymatic degradation protects neurons from glutamate excitotoxicity. J Neurochem. 2000;75(3):1045-1052.

53. Needham LL, Grandjean P, Heinzow B, et al. Partition of environmental chemicals between maternal and fetal blood and tissues. Environ Sci Technol. 2011;45(3):1121-1126.

54. McGaugh JL. Making lasting memories: remembering the significant. Proc Natl Acad Sci USA. 2013;110(suppl 2):10402-10407.

55. Wang J, Zhang Y, Guo Z, et al. Effects of perinatal fluoride exposure on the expressions of miR-124 and miR-132 in hippocampus of mouse pups. Chemosphere. 2018;197:117-122.

56. Liu F, Ma J, Zhang H, et al. Fluoride exposure during development affects both cognition and emotion in mice. Physiol Behav. 2014;124:1-7.

57. Bhatnagar M, Rao P, Saxena A, et al. Biochemical changes in brain and other tissues of young adult female mice from fluoride in their drinking water. Fluoride. 2006;39(4):280-284.

58. Anderson LM, Diwan BA, Fear NT, Roman E. Critical windows of exposure for children's health: cancer in human epidemiological studies and neoplasms in experimental animal models. Environ Health Perspect. 2000;108(suppl 3):573-594.

59. Basha PM, Rai P, Begum S. Evaluation of fluoride-induced oxidative stress in rat brain: a multigeneration study. Biol Trace Elem Res. 2011;142(3):623-637.

60. Basha PM, Sujitha NS. Combined impact of exercise and temperature in learning and memory performance of fluoride toxicated rats. Biol Trace Elem Res. 2012;150(1-3):306-313.

61. Khan AM, Raina R, Dubey N, Verma PK. Effect of deltamethrin and fluoride co-exposure on the brain antioxidant status and 
cholinesterase activity in Wistar rats. Drug Chem Toxicol. 2018; 41(2):123-127.

62. Thierry AM, Glowinski J, Goldman-Rakic PS. Motor and Cognitive Functions of the Prefrontal Cortex. Springer: Research and Perspectives in Neurosciences. 2012.

63. Pisa M, Sanberg PR, Fibiger HC. Locomotor activity, exploration and spatial alternation learning in rats with striatal injections of kainic acid. Physiol Behav. 1980;24(1):11-19.

64. Lago T, Davis A, Grillon C, Ernst M. Striatum on the anxiety map: small detours into adolescence. Brain Res. 2017;1654(pt B): 177-184.

65. Bannerman DM, Sprengel R, Sanderson DJ, et al. Hippocampal synaptic plasticity, spatial memory and anxiety. Nat Rev Neurosci. 2014;15(3):181-192.

66. Zhu YP, Xi SH, Li MY, et al. Fluoride and arsenic exposure affects spatial memory and activates the ERK/CREB signaling pathway in offspring rats. Neurotoxicology. 2017;59:56-64.
67. Desai SN, Desai PV. Aspartate aminotransferase and alanine aminotransferase activities of rat brain during crush syndrome. $\mathrm{Neu}$ rosci Lett. 2008;447(1):58-61.

68. Blaylock RL. Excitotoxicity: a possible central mechanism in fluoride neurotoxicity. Fluoride. 2004;37(4):301-314.

69. Shivarajashankara YM, Shivashankara AR. Neurotoxic effects of fluoride in endemic skeletal fluorosis and in experimental chronic fluoride toxicity. J Clin Diagnostic Res. 2012;6(4): 740-744.

70. Bai X, Zhang C, Chen A, et al. Protective effect of edaravone on glutamate-induced neurotoxicity in spiral ganglion neurons. Neural Plast. 2016;2016:4034218.

71. Cattani D, Cesconetto PA, Tavares MK, et al. Developmental exposure to glyphosate-based herbicide and depressivelike behavior in adult offspring: implication of glutamate excitotoxicity and oxidative stress. Toxicology. 2017;387: $67-80$. 